\title{
Tangence
}

\section{Le genre comme procès axiologique et esthétique : éléments pour l'enseignement du roman africain}

\section{Josias Semujanga}

Numéro 49, décembre 1995

Les littératures francophones de l'Afrique et des Antilles

URI : https://id.erudit.org/iderudit/025880ar

DOI : https://doi.org/10.7202/025880ar

Aller au sommaire du numéro

Éditeur(s)

Tangence

ISSN

0226-9554 (imprimé)

1710-0305 (numérique)

Découvrir la revue

Citer cet article

Semujanga, J. (1995). Le genre comme procès axiologique et esthétique : éléments pour l'enseignement du roman africain. Tangence, (49), 94-111.

https://doi.org/10.7202/025880ar d'utilisation que vous pouvez consulter en ligne. 


\title{
Le genre comme procès axiologique et esthétique : éléments pour l'enseignement du roman africain
}

Josias Semujanga

\author{
C'est par le refus et la transgression \\ que les genres modernes se consti- \\ tuent.
}

Dominique Combe ${ }^{1}$

\section{Introduction méthodologique}

La question des genres littéraires dans l'enseignement de la littérature africaine en général et du roman en particulier est, de Mohamadou Kane à Ambroise Kom en passant par Bernard Mouralis $^{2}$, l'objet central de la critique ${ }^{3}$. Pourtant, pendant plusieurs années la méthode historiciste et sociologique des ouvres, qui avait été longtemps privilégiée, avait fini par escamoter le débat. Maintenant que le renouvellement de la théorie littéraire a entraîné une reformulation de la question des genres, il s'agit de

1 Dominique Combe, Les genres littéraires, Paris, Hachette, 1992, p. 157.

2 Mohamadou Kane, "L'enseignement de la littérature africaine en Afrique", Colloque Littératures africaines et enseignement, Bordeaux, Presses universitaires de Bordeaux, p. 27-39; Bernard Mouralis, "L'université a-t-elle peur de la littérature africaine d'expression française?", numéro spécial - Littératures africaines et universités françaises, Recherche, pédagogie et culture, $n^{\circ} 57$, 1982, p. 4-9; Ambroise Kom, "Francophonie et enseignement des litteratures africaines: quels enjeux?", Revue francopbone, vol.vIII, $\mathrm{n}^{\circ} 1$, p.103-109. Sur les littératures francophones en général, on peut se reporter au numéro spécial d'Itinéraires et contacts de cultures: L'enseignement des littératures francophones, Paris, L'Harmattan, 1982.

3 Pour des raisons qui tiennent essentiellement à la nature de cet article, je n'aborclerai pas des questions qui touchent aux rapports entre un texte et la nationalité de l'écrivain. Qu'est-ce qui établit la nationalité d'un texte ou d'un groupe de textes? Est-ce la nationalité de l'écrivain qui définit celle de son ceuvre? Toutes ces questions et d'autres du même type ne seront pas prises en compte ici. Je mettrai plutôt en valeur l'idée selon laquelle les textes africains à la manière d'autres textes modernes sont caractérisés par une activité générique très poussée de telle sorte que les classifications par genres littéraires sont très difficiles à établir. 
montrer, à partir d'un double regard sur le genre comme procès axiologique et esthétique que, d'une part le roman africain participe pleinement des pratiques littéraires contemporaines, caractérisées par un éclatement majeur des genres, et que d'autre part la notion de genre reste centrale dans l'enseignement des textes littéraires et surtout des textes africains qui sont marqués de façon significative par une esthétique polygénérique. La convergence dans le concept de genre, de traits formels de nature aussi différente que des motifs du conte africain, du roman policier occidental, etc., en fait un objet d'investigation pour toute critique qui s'attache aussi bien à la signification anthropo-sociologique qu'à la dimension esthétique d'une ouuvre littéraire ${ }^{4}$.

Par ailleurs, cette ambiguité inhérente au concept de genre luimême est liée également au paradoxe primordial de tout discours littéraire qui, sur le plan narratif mobilise les mécanismes du genre, alors que, sur le plan sémantique, il est un discours situé ayant une forte efficacité communicative portant, notamment, sur l'évaluation des pratiques sociales. C'est donc un double procès axiologique et esthétique. Pour le roman, comme l'affirme Pierre-Louis Rey, ce double procès est sémantiquement lié au mot "romanesque" luimême dont l'ambiguité fait qu'il "désigne tantôt une manière d'être, tantôt un genre littéraire " ${ }^{5}$. En paraphrasant Milan Kundera, l'on pourrait dire que l'un des traits majeurs du genre romanesque, c'est justement d'être un carnaval de la relativité morale ${ }^{6}$. En effet, dans

4 Beaucoup de critiques ont montré l'intérêt de centrer l'étucle du roman africain sur les rapports que celui-ci établit avec la littérature orale traditionnelle. Entre autres études, on peut citer celles d'Eileen Julien, African Novels and the Question of Orality, Bloomington/Indianapolis, Indiana University Press, 1992 et d'Amadou Koné, Des textes oraux au roman moderne. Étude sur les avatars de la tradition orale dans le roman ouest-africain, Franfurt, Verlag fur Interkulturelle Kommunikation, 1993. Mais l'esthétique du roman africain ne saurait être réduite à cette seule dimension. S’il est vrai que la convocation des traits génériques de l'oralité africaine est une dominante dans certains textes, il est rare que l'intertextualité liant le roman africain à d'autres romans modernes soit absente. C'est pourquoi cette multiplicité des traits génériques, loin de constituer une spécificité du roman africain, situe celui-ci plutôt dans les préoccupations esthétiques du roman moderne en général, de Marquez à Chamoiseau en passant par Sollers ou Kafka.

5 Pierre-Louis Rey, Roman et bistoire, Paris, Hachette, 1992, p. 3.

6 Milan Kundera utilise ce terme en parlant du roman de Rushdie, Les versets sataniques: "[...] personne n'a raison et personne n'a entièrement tort dans cet immense carnaval de la relativité qu'est cette ceuvre». $C f$. Milan Kundera, Les testamments trabis, Gallimard, 1994, p. 40. 
96

la mesure où l'on peut considérer le texte littéraire comme un discours, sa lecture se présente comme une mise en place d'une situation de communication portant généralement sur des pratiques sociales à partir desquelles les interlocuteurs établissent entre eux un contrat d'échange de type axiologique, explicite à la fois dans le choix des pratiques qui sont socialement réglées - c'est-à-dire soumises à des évaluations - et leur propre position axiologique sur ces pratiques. Comment un texte produit-il une valorisation? Sur quoi porte-t-elle? L'hypothèse est que le récit littéraire prend sa position axiologique à partir d'une réflexion sur les fonctions narratives des traits génériques dans les textes. En ce sens le genre fonctionne comme une pratique littéraire conventionnellement réglée permettant l'évaluation d'une ceuvre, c'est-à-dire le lieu où s'effectue le double procès axiologique et esthétique d'un texte. Il ne saurait être question de présenter ici un relevé complet de toutes les pratiques socialement réglées sur lesquelles peut porter une lecture axiologique des textes. On peut signaler néanmoins trois hypothèses pour l'écriture romanesque: (1) à travers l'histoire qu'il raconte, tout roman tient un discours sur le processus de création littéraire lui-même; (2) celui-ci comporte une dimension de valorisation (jugements de conformité ou de non conformité, de positivité ou de négativité, etc.) et (3) c'est à partir des motifs narratifs qui lui sont propres que le roman intègre d'autres traits formels spécifiques à d'autres genres littéraires. C'est également par là qu'il brasse et oppose en son sein nombre de systèmes de valeurs différents et pose son propre point de vue sur l'écriture.

$\grave{A}$ partir de trois romans africains - Doguicimi ${ }^{7}$, Le devoir de violence ${ }^{8}$ et Shaba deu $x^{9}$ - il s'agit de montrer que les parcours narratifs des principaux personnages se construisent à partir de la confrontation polémique de type moral et que leur neutralisation par l'ambivalence axiologique est lié à une prise de position sur la création romanesque elle-même. L'analyse se fera en trois temps: (1) les parcours narratifs des principaux acteurs des récits, (2) leur ambivalence axiologique et (3) la neutralisation du point de vue moral comme principe de création romanesque.

7 Paul Hazoumé, Doguicimi, Paris, Maisonneuve et Larose, 1938.

8 Yambo Ouologuem, Le devoir de violence, Paris, Seuil, 1968.

9 Valentin Yves Mudimbe, Shaba deux. Les Camets de Marie-Gertrude, Paris, Présence africaine, 1989. 


\section{De l'absurde comme procès axiologique et esthétique ${ }^{10}$ dans Shaba deux}

À première vue, rien, même pas sa date de parution, 1989, n'autorise le choix d'un roman comme Shaba deux pour tenter, à partir de ce qu'il donne à lire, de parler de la réutilisation du thème de l'absurde. Au plus fort de la guerre du Shaba, Sœur Marie-Gertrude est nommée Mère supérieure d'un couvent en désintégration, car il est déserté par ses consœurs européennes qui quittent la ville sous la protection militaire dépêchée sur les lieux par l'ancienne métropole. Restée seule à s'occuper du couvent, dont elle est désormais l'unique locataire franciscaine, des malades et des blessés, Marie-Gertrude sera assassinée par les soldats de l'armée gouvernementale. Jeté dans la rivière, son cadavre mutilé sera découvert par deux adolescents qui pêchaient dans la rivière Lualaba. Une telle fin ne contredit-elle pas le télos humaniste que, par ailleurs, le roman déploie? Dans ce cas, peutil y avoir véritable révélation d'un sens de la vie? La quête du sens, qui se double de la quête du genre, prend ici une inquiétude devant la "déchirure" d'une héroïne qui tente d'opposer à l'absurde, qui régit son univers relationnel avec ses consceurs franciscaines, un idéal et un style de vie différents ${ }^{11}$.

Le parcours narratif de Sœur Marie-Gertrude en tant que sujet de l'absurde fonctionne comme un cercle vicieux. Tout commence avec un long cheminement spirituel et moral qui a conduit l'hérö̈ne dans les ordres des franciscaines. Son projet est de continuer le témoignage d'amour, de générosité et d'abnégation hérité de Sœur Angélique, son ancien professeur de littérature au lycée, morte noyée:

J'ai rencontré Sœur Angélique lorsque j'étais en troisième grécolatine. Une femme remarquable: jeune, ouverte, et surtout, oh surtout sa générosité. Elle m’a donné le goût de la littérature et le sens de la beauté. Notre vie, me disait-elle, devrait être parfaite et lumineuse à l'instar d'une période cicéronnienne [...] Une voix pure. Une lame de lumière. Elle était belle. Je fus

10 Sur l'absurde comme principe d'écriture, voir l'article de Jacques Fontanille, "L'absurde comme forme de vie", Recherches sémiotiquessemiotic Inquiry, vol. XIII, nos 1-2, 1994, p. 95-116.

11 À la lecture des textes de Mudimbe se dégage, en effet, une idée permanente, presque un leitmotiv: la nécessité impérieuse d'inventer un style de vie nouveau qui permette de repenser la vie à partir d'une situation historique: la colonisation occidentale en Afrique. 
98

transpercée sur le coup. Je décidai d'être comme elle. Pour rayonner d'une passion aussi vibrante. Et quand, une année plus tard, au retour des vacances de Noël, on nous apprit qu'elle s'était noyée, je me promis, dans le silence de mon cour, de devenir franciscaine afin de continuer son témoignage ${ }^{12}$.

À la mort de son professeur, Sœur Marie-Gertrude prend conscience de l'existence dans le monde de deux ordres contradictoires: le bonheur recherché par l'être humain et l'injustice de la mort. De ce vide existentiel, elle recherche une compensation en allant au couvent des franciscaines de Kolwezi. La déception est totale. Elle ne retrouve pas la plénitude de l'existence qu'elle cherche. La compensation dans les prières et la vie paisible ne la satisfont pas, non plus. La quête du sens se transforme ainsi en angoisse du dérisoire à laquelle l'hérö̈ne aboutit. Elle se révolte pour donner sens à ses actions. Dans un troisième temps, elle recherche une nouvelle compensation dans la complémentarité de la prière et du travail social. Là aussi son échec ramène le parcours narratif à la phase de départ, au manque initial de l'héroïne. Le serpent se mord la queue dans l'angoisse du dérisoire, qui débouche sur la mort de l'héroïne comme fin des illusions. Ce parcours narratif, reconstitué après coup puisque le récit refuse la linéarité de l'histoire, crée un vide faisant voir un récit orienté vers l'aventure "humaniste" et vers la quête existentielle d'une identité problématique. L'héroine est en quête d'une morale pour donner sens au non-sens de sa vie.

Comme principe narratif, l'absurde résulte de l'ambivalence morale des actions des principaux personnages: Mère Laetitia et Sœur Marie-Gertrude ${ }^{13}$. De passion nulle dans sa dimension

12 Valentin Yves Mudimbe, op. cit., p. 36-37.

13 Ce principe de la neutralisation des points de vue moraux fonctionne comme un leitmotiv dans tous les romans de Mudimbe. Dans Entre les eaux, Paris, Présence africaine, 1973, Pierre Landu est un prêtre qui oscille entre la foi catholique et la révolution marxiste. Jusqu'à la fin du récit, le héros ou plutôt l'antihéros navigue entre les deux courants. Nara de L'écart, Paris, Présence africaine, 1979 , ne parvient pas à choisir entre les valeurs de l'Occident et celles de l'Afrique précoloniale. Il meurt écartelé entre les deux univers culturels. Il est marginalisé par les autres protagonistes. Il est à l'écart, dans l'intersection de deux ensembles culturels. Dans Le bel immonde, Paris, Présence africaine, 1976, il s'agit d'une mise en scène d'un couple profondément ambivalent quant aux objets de valeur respectifs poursuivis. Dans la mesure où elle rythme le tempo de la narration romanesque chez Mudimbe, l'ambivalence axiologique semble ainsi se transmuer en procès esthétique. 
axiologique, l'absurde se transforme en procès esthétique: le sujet de l'absurde acquiert une liberté créatrice. Le récit donne alors plusieurs pistes de réutilisation de l'absurde comme principe d'écriture. On en retient quatre qui fonctionnent comme des stratégies de l'écrivain. D'abord, le narrateur convoque des pratiques socialement réglées à travers un genre littéraire - le journal intime - et un thème - la guerre du Shaba - et construit un système de modèles ou de repoussoirs qui permettent au lecteur de situer la source d'énonciation du texte et de son système évaluatif. Ainsi, le personnage de Mère Lætitia, Supérieure du couvent franciscain de Kolwezi représente la morale du "devoir", c'est-à-dire celle qui consiste à suivre des normes institutionnelles, en l'occurrence des préceptes de l'ordre des franciscains. En se situant en faux contre cette morale, Sour Marie-Gertrude apparaît, à travers son ironie, comme représentante d'un autre type de morale: celle du sujet de l'absurde, qui, par son refus de l'ordre des valeurs établies, cherche des valeurs de remplacement en pratiquant la morale du "refus". Ensuite, la polarisation axiologique du narrateur sur Marie-Gertrude montre que celle-ci est le personnage qui incarne un système de valeurs marqué comme positif. Elle est, en effet, le centre de l'évaluation du projet missionnaire de l'Église catholique en Afrique. À la morale du "devoir", qui s'appuie sur les dogmes de l'Église pour soutenir la spiritualité, elle oppose une éthique de l'action qui va à l'encontre de la morale du "devoir" prônée par sa congrégation. Malgré sa morale du "refus", Marie-Gertrude ne sera pas moins fauchée par la répression militaire, symbole du mal absolu qui frappe tout le monde. Dès le départ, elle se sait condamnée. En attendant l'issue fatale, elle fait ce qu'elle peut pour diminuer les douleurs des malades et des blessés de guerre. Elle pratique, à l'instar du $\mathrm{D}^{\mathrm{r}}$ Rieux de La peste, la morale de la mesure qui consiste en la conquête du possible et le refus de capituler sans être sûr du lendemain ${ }^{14}$. En troisième lieu, la stratégie du narrateur, qui est d'ordre syntagmatique, consiste à désambiguiser, à la fin du récit, le texte en évaluant les réussites et les échecs des parcours narra-

14 Cette morale de la conquête du possible est celle que l'on retrouve dans l'ouvre d'Albert Camus, notamment Le mythe de Sisyphe (Paris, Gallimard, 1943) et La peste (Paris, Gallimard, 1947). Voir, à ce propos, une étude inédite de Josias Semujanga sur la dialectique de l'absurde chez Camus : La problématique du mal et l'bumanisme dans La peste d'Albert Camus, Bujumbura, Université du Burundi, mémoire de maitrise, 1986. 
tifs des personnages. Ainsi, si la mort de Marie-Gertrude marque symboliquement les limites de la théologie africaine, qui entend réconcilier spiritualité et action sociale, la sanction négative de son parcours narratif est aussi liée à une autre évaluation: séparer Dieu de la pratique religieuse comme le précise Sour MarieGertrude à Sour Marie-Cécile, Mère Supérieure des Novices: "[...] Et puis, je vous prie, laissons Dieu hors de tout ceci, et faisons face à notre condition d'êtres humains parmi d'autres humains que la bêtise, la convoitise, la méchanceté, un goût immodéré du pouvoir condamnent; écrasent, tuent [...]"15. Enfin, la dernière ruse a consisté à disjoindre l'évaluation positive de l'évaluation négative. Ainsi, le projet de Mère Latitia de créer un couvent où les membres vivent en prière loin du monde est présenté explicitement comme positif selon la morale du "devoir" franciscain pour signifier sa négativité selon l'évaluation qu'en fait la narratrice et sa morale du "refus". Inversement, le projet de MarieGertrude d'être missionnaire parmi les siens et de réconcilier spiritualité et action sociale est présenté positivement alors que la solitude finale de l'héroine et sa mort tragique signifient implicitement la négativité du projet: la dénégation de l'action comme source de salut de l'homme. Le récit neutralise ainsi les deux points de vue moraux, qu'il a, par ailleurs longtemps séparés.

La neutralisation du point de vue ou l'ambivalence des parcours axiologiques des personnages amène la narratrice à adopter à la fois les techniques du journal intime, de la narration romanesque et de l'essai. L'instabilité et la réversibilité des significations, qui sont liées à la découverte du dérisoire à l'intérieur des systèmes moraux contradictoires, n'autorisent pas qu'il y ait une véritable rêvélation d'un sens. Une telle quête, déceptive par essence, devient alors aussi celle du genre: le roman. En effet, Shaba deux peut se lire sur plusieurs portées comme une partition musicale. Il

15 Valentin Yves Mudimbe, op. cit., p. 137. L'épisode de la guerre civile constitue le moment crucial dans le parcours narratif de Marie-Gertrude. Il permet au narrateur de poser un jugement moral sur des actes posés par les personnages. C'est à partir du problème de Dieu que le récit dévoile sa position morale. Ce roman rappelle, par ailleurs, la position de Jean-Paul Sartre concernant le problème de Dieu et du rappon avec autrui : "Le problème de Dieu est un problème humain qui concerne le rapport des hommes entre eux, c'est un problème total auquel chacun apporte une solution par sa vie entière, et la solution qu'on lui apporte reflète l'attitude qu'on a choisie vis-àvis des autres et de soi-même", Jean-Paul Sartre, Situations IV, Paris, Gallimard, 1964, p. 88. 
est le récit d'un journal intime qui livre des réflexions personnelles d'un personnage sur divers sujets. C'est aussi une analyse des réactions collectives devant la guerre. Il peut se lire aussi comme un essai éthique sur l'attitude de l'Église face aux problèmes sociaux. Sobre et tenue, l'écriture de Shaba deux est un art du "contrepoint romanesque [...] susceptible de souder en une seule musique la philosophie, le récit et le rêve, [...] un essai spécifiquement romanesque qui ne prétende pas apporter un message apodictique mais reste hypothétique, ludique, ou ironique "16. Si Shaba deux peut, entre autres, se lire comme l'histoire manquée d'une révolution morale et l'échec de la conquête de l'être, elle est, par ailleurs, le lieu d'assomption sémantique de l'absurde. De passion inutile, quand celle-ci signifie quête impossible de l'absolu de l'être, l'absurde devient exercice de la liberté créatrice et principe de la narration. Enseigner Shaba deux implique ainsi au moins trois choses: (1) Ce texte montre que la notion de genre est moins absolue qu'on ne croit, car il intègre aux procédés romanesques les traits génériques de la réflexion essayistique; (2) par la convocation de plusieurs traits génériques, il participe ainsi de l'esthétique du roman contemporain et (3) son parcours thématique et sa suspension du point de vue moral le rattachent davantage au roman européen dit de l'absurde que des grands topoï du roman africain. D'autres romans africains comme Le devoir de violence, par exemple, fonctionnent selon les mécanismes semblables: l'ambivalence axiologique et la multiplicité des traits génériques.

\section{Ambivalence axiologique et transformation narrative dans Le devoir de violence}

Participant de la légende, de l'épopée, de la chronique et du roman, Le devoir de violence est un texte difficile à classer dans un genre précis à tel point que les critiques se lancent la balle à propos des sources du roman. Pour les uns, l'auteur a plagié les romans occidentaux tandis que pour d'autres le roman trouve ses sources dans les cultures africaine. Ce qui prouve tout simplement que ce roman navigue à contre-courant de la rectitude générique et identitaire. En effet, le roman est divisé en quatre parties. Dans la première, intitulée "La légende des Saifs", le narrateur raconte sur le mode parodique le passé de l'Afrique

Milan Kundera, L'art du roman, Paris, Gallimard, 1986, p. 83. 
102

précoloniale symbolisée par le royaume imaginaire du Nakem. La deuxième raconte de façon ironique l'histoire de la résistance des Saifs à la colonisation française. La troisième partie raconte la vie dure faite, à la cour impériale, aux serfs et aux captifs. Dans ce tableau sombre se détache un couple amoureux formé du cuisinier Kassoumi et de la servante Tambira. De leur mariage naitront des quintuplés dont Raymond Spartacus Kassoumi qui ira faire ses études à Paris avant de devenir député de son pays à l'assemblé nationale française. Ayant créé un réseau d'espions, Saïf tue par empoisonnement des gouverneurs français (Chevalier et Vandame) et d'autres opposants. Seul un évêque, Henry, déjoue ses stratégies. Et la dernière partie, qui est une sorte d'épilogue "L'aurore ", se termine sur un motif du jeu. Conscient de se précipiter dans la gueule du loup, l'évêque Henry se présente chez Saif. Les deux hommes en viennent à discuter de la violence, de l'amour et de Dieu avant de jouer aux échecs, alors que tangue entre eux une flûte contenant une vipère aspic que Saif destinait à l'évêque mais qu'il jette plus tard au feu. Le mérite de ce roman se trouve dans ce motif du jeu entre différents textes et différents genres littéraires de l'Afrique et de l'Europe.

Une telle transformation des traits génériques existants s'accompagne de l'ambivalence axiologique des personnages. Liée, par ailleurs, au principe de parodisation qui rythme les parcours narratifs du roman, l'ambivalence axiologique du texte se fait en trois temps. D'abord, l'ironie du narrateur incorpore au roman des allusions directes aux textes fondateurs de la négritude, de l'ethnologie et de la colonisation sur lesquelles les personnages prennent position dans leur évaluation morale. En deuxième lieu, les personnages de Saif, Shrobénius sont le lieu des polarisations négatives tandis que ceux de Kassoumi et Henry sont investis des valeurs positives. En dernier lieu, le narrateur suspend son système de jugement moral par la réconciliation de valeurs contradictoires de ces personnages; ce qui rend ambivalent le comportement moral de chaque parcours narratif. Autant Saif et son alliê Schrobénius sont le symbole de la destruction de l'Afrique, autant Kassoumi et l'évêque Henry incarnent l'espoir de l'Afrique. C'est à partir de ces personnages que se fait le procès axiologique du roman ${ }^{17}$. Malgré son caractère de bourreau san-

$17 \mathrm{Si}$, à ce propos, le roman se compare à bien d'autres, son originalité réside sans doute dans la psychologie très dense des personnages dont le caractère 
guinaire sur lequel focalise la narration, Saif apparaît sous certains aspects comme un héros. N'a-t-il pas su déjouer les stratégies de ses ennemis? Par moments, le narrateur neutralise cette évaluation ironique qui a chargé de traits négatifs le roi Saif et l'ethnologue Shrobénius. On assiste alors à une description méliorative du personnage du roi qui passe de la bassesse roturière à la magnanimité princière. Même s'il voue une haine ouverte au sorcier Bourémi, le fou du village, Saif adopte l'enfant de ce dernier laissé par sa femme morte en couches ${ }^{18}$. Il en est de même de Shrobénius qui, voulant ressusciter les valeurs africaines enfouies dans les masques, poursuit dans une certaine mesure un projet sans intérêt et se fait complice de Saif tout en faisant montre d'une générosité sans faille à l'égard du continent noir.

De même certains épisodes du roman renvoient dos à dos le roi Saiff et le peuple. Certes le peuple se rachète dans la figure de Raymond Kassoumi, fils des esclaves Kassoumi et Tambira. Celuici refuse de collaborer avec Saif, qui a vendu son père après avoir tué ses frères, mais accepte de rentrer au pays natal pour être député. Même s'il s'inscrit dans une mythologie du peuple, Kassoumi est loin d'être un saint. À Paris, il a commis l'inceste avec sa sœur; il s'est prostitué afin de survivre après la suspension de sa bourse d'études et il a trompé sa femmé quand celle-ci était enceinte. Issu de modeste condition, ce personnage incarne malgré tout l'espoir de l'Afrique et symbolise celui du peuple. Il est aidé par l'évêque Henry, le seul personnage blanc qui a refusé de collaborer avec Saif et l'autorité coloniale. Son caractère velléitaire lui enlève cependant la stature de héros, car il n'incarne pas vraiment des valeurs hautement positives pour susciter l'adhésion du lecteur. L'ironie et le paradoxe bien maniés permettent ainsi au narrateur de brasser et d'opposer, à partir de ces figures emblématiques nombre de valeurs différents; cela permet en même temps de suspendre le système évaluatif du roman ${ }^{19}$.

profondément ambivalent rend possible cet art du contre point romanesque que le roman pratique avec efficacité : le refus de constituer la narration à partir d'un point de vue privilégié.

18 Yambo Ouologuem, op. cit., p. 99.

19 Ces personnages échappent ainsi au schéma manichéen du méchant puni et du bon récompensé auquel le roman africain semble avoir habitué le lecteur comme je l'ai déjà montré dans une autre étude : "Tout se passe comme si la narration mettait en cause le caractère fonctionnel et dénotatif de ce roman 
104

En mettant en parallèle les procédés narratifs, les parcours narratifs et les plans sémantiques, on constate que l'innovation du Devoir réside dans ce double procès axiologique et esthétique qui, en fusionnant divers traits génériques et en les multipliant incessamment, les renverse tous. En intégrant aux procédés narratifs du roman l'esprit du non-sérieux et du ludique, Le devoir a non seulement renversé les dogmes du réalisme, qui prétend suggérer au lecteur l'illusion du réel, mais a aussi reconquis le droit à la transgression des genres comme mode privilégié par le narrateur pour l'esthétique romanesque. Cette écriture d'un non-lieu axiologique $^{20}$ et générique fait de ce roman l'un des plus grands textes de la littérature africaine contemporaine dont l'écriture polythêiforme convoque à la fois les traits génériques des textes africains, européens ou latino-américains.

\section{L'art du contre point romanesque dans Doguicimi}

Cet art du contrepoint romanesque se construit par la fusion dans la narration des textes de chansons inspirés des récits oraux et des traits formels du roman historique. Malgré l'importance accordée par le narrateur aux descriptions à caractère anthropologique et ethnologique, l'histoire est davantage un cadre de lecture, un arrière-plan à l'intrigue qu'un but recherché pour luimême. Aux personnages reconnus par la tradition historique sont adjoints des personnages purement imaginaires - comme Doguicimi qui a donné son nom au roman - et aux événements enregistrés par l'histoire se substituent souvent des événements fictifs.

et permettait de détruire le cliché du discours négro-africain et ses avatars ethnologiques; ce qui crée un affrontement entre l'écrivain et ce que la communauté attend de lui. Ce faisant, Ouologuem revendique le droit d'écrire sans être porte-parole. L'ironie, la satire et la parodie déstabilisent les conventions et les topoï du roman réaliste africain et attirent l'attention sur les mécanismes d'écriture dont l'ambivalence sémantique est le mode privilégié dans le devoir. Cela situe ce texte dans les grandes préoccupations esthétiques du roman du $x^{\mathrm{e}}$ siècle $[. .$.$] ". C f$. Josias Semujanga, "De l'histoire à sa métaphore dans Le devoir de violence de Yambo Ouologuem", Études françaises, vol. XXXi, no 1,1995 , p. 81).

20 Cette ambivalence axiologique fait que le narrateur ne privilégie aucun point de vue. Le devoir se dérobe à une lecture monologique, car le narrateur-ironiste "renvoie dos à dos toutes mythologies en cours en Afrique : celle de l'Eldorado précolonial, celle du Blanc "civilisateur" et celle du marxisme postcolonial. Il s'agit d'une mise en écriture de la polyphonie discursive et d'un non-lieu idéologique ". Ibid., p. 81. 
Dès lors qu'il fonctionne comme une opération intersémiotique entre le roman et le contexte référé, le discours de savoir ethnologique ou historique, est moins une représentation de la réalité extra-fictionnelle qu'un mode conventionnel de lecture du roman. Tourné vers l'organisation interne - configuration générale des actions, des personnages et des figures de rhétorique - la narration de Doguicimi déstabilise le processus explicatif du discours anthropologique. Car, tout en racontant le monde qui l'entoure - son extériorité - le roman est aussi une réflexion sur ce qu'est l'écriture littéraire à partir d'une histoire d'amour d'un intérêt romanesque remarquable. Le thème central du roman n'est-il pas en effet la constance héroïque de Doguicimi, princesse d'Abomey, dont l'époux, le prince Toffa, meurt chez les Mahinous? Doguicimi est une princesse qui symbolise la fidélité dans ce Dahomey du $\mathrm{XIX}^{\mathrm{e}}$ siècle. Certes, le sacrifice par lequel elle se fit enterrer vivante est historiquement vrai, mais, à travers cet acte héroïque, Doguicimi est liée à un parcours narratif plus global, qui consiste à dégager une figure plus complexe de la femme dans les rapports avec son foyer, son mari et ses coépouses, puis dans ses rapports avec la cour et la politique.

Très belle femme, décrite avec sensualité, Doguicimi fait l'admiration de tous. Son mari, Toffa, ne lui donne-t-il pas justement un nom très motivé qui signifie "distinguez-moi "? Toffa est comblé par une femme qui sait lui parler doucement d'amour. C'est à ce moment, au premier chapitre, que sept vers de chanson à dominante thématique de guerre sont insérés dans le texte. Au deuxième chapitre un autre texte de chanson annonce l'oracle qui prédit la défaite des Dahoménous. Cette transformation chantée cause de l'angoisse chez tout le monde, mais surtout chez le couple Doguicimi-Toffa ${ }^{21}$. Toffa est chef des armées et ses responsabilités militaires et politiques l'accaparent. Il se trouve devant un dilemme. Patriote et prince, il est prêt à tout pour défendre son pays; or, il juge insensée la guerre que projette le roi contre son voisin mahinou. Il trouve que faire la guerre pour venger deux Européens tués par les Mahinous n'est pas un motif sérieux. C'est ainsi que Toffa tente de s'opposer sans succès au danger que représentent les "troncs blancs" "qui incarnent "des choses opposées à nos mours, à nos coutumes" "22. Malgré cela

21 Paul Hazoumé, op. cit., p. 74.

22 Ibid., p. 44. 
106

Toffa rejoint l'armée et laisse sa femme seule et désespérée. Au troisième chapitre, l'invincibilité des Dahoménous, chantée par la reine sur le champ de bataille, prépare le coup de théâtre que constitue la nouvelle de la défaite, déjà annoncée par l'oracle de la chanson du premier chapitre ${ }^{23}$.

Au quatrième chapitre le texte de chanson devient dense. Il est une explication de la défaite et dans l'ensemble du récit, il est lié à une autre transformation: la préparation de la revanche des Dahoménous contre les Mahinous ${ }^{24}$. Au cinquième chapitre, au moment de la guerre de revanche, Doguicimi doit affronter les difficultés inhérentes à sa condition de femme seule, car, en l'absence de son mari, sa beauté lui attire bien des ennuis. À la jalousie de ses coépouses s'ajoute le désir des hommes que suscite sa beauté. Elle jure fidélité à son mari en composant un hymne dont le propos se situe dans le nouveau parcours thématique de cette séquence narrative: la revanche. Les deux chants qu'elle compose constituent une réponse à l'oracle et au dialogue entre elle et son mari au premier chapitre. Du sixième au huitième chapitres, les chansons célèbrent la force du roi Guézo, roi du Dahomey, et mobilisent la jeunesse pour la revanche contre les Mahinous.

$\mathrm{Au}$ neuvième chapitre, les textes de chanson s'attardent sur le personnage de Doguicimi. Un changement de focalisation important s'est opéré. Car depuis le deuxième chapitre le double problème qui s'était posé n'était pas encore résolu: la revanche des Dahoménous contre les Mahinous et le sort du couple DoguicimiToffa. Maintenant que les Mahinous sont vaincus, le narrateur se concentre sur Doguicimi. Les deux derniers couplets du texte de chanson de cette séquence narrative vont dans ce sens. La première chanson relance le débat sur le choix que devra faire Doguicimi : accepter ou refuser les avances du prince héritier, Vidaho et en assumer les conséquences ${ }^{25}$.

Vidaho s'éprend en effet de Doguicimi, mais celle-ci refuse de l'épouser. Éconduit, Vidaho charge sa cousine, Noucouzin, de la délicate mission de convaincre l'insoumise. Des pratiques magiques pour envoûter Doguicimi et la ruse ayant échoué, Nou- 
couzin recourt sans succès à la menace: "Femme du peuple, tu n'es qu'un oiseau qui s'est posé accidentellement sur cet arbre " 26. C'est alors que le complot est envisagé. Doguicimi est accusée d'avoir fait des avances à Zambounou, l'esclave mahinou dont le pays a attaqué le Dahomey. Son refus du compromis en fait une héroïne contre ses coépouses et autres courtisanes aux mœurs légères "persuadées que la femme qui, dans la vie, n'aura connu qu'un seul homme sera honnie dans l'empire des morts"27. C'est dans cette épreuve que se révèle la grandeur de Doguicimi. Fouettée par le bourreau, jetée au cachot, elle endure un véritable martyre. En plus de la douleur physique, Doguicimi est en proie à une profonde déchirure psychologique. Elle se demande si Toffa, son mari, l'aimait vraiment puisqu'il ne lui a donné aucun enfant.

La dernière chanson, dite par l'héroïne, a le rythme d'une marche funèbre. Le roman tire alors à sa fin. La décision de se donner la mort plutôt que de céder à Vidaho ou à ceux qui la tourmentent provient-elle d'abord du vou de Doguicimi de rester fidèle à son mari malgré tout, ou plus subtilement d'un appel irrésistible au suicide? Le narrateur ne tranche pas. Tant mieux pour le lecteur! Et cette ambiguité, loin d'être déroutante constitue l'originalité de ce texte. L'énonciation convoque toutes les valeurs et les neutralise. N'est-ce pas là le propre de l'œuvre littéraire en général et du roman en particulier d'être le lieu d'assomption de tout ce qui reste inexprimé et inexprimable par le langage quand on met le monde et les mots pour le traduire ensemble comme le dit justement Hermann Broch: "Le but, infini, jamais atteint de la science: obtenir une image totale de la connaissance, le désir infini, jamais exaucé, des systèmes particuliers de valeurs : parvenir à l'absolu et réaliser une union entre tous les éléments rationnels et irrationnels de la vie, tout cela trouve un accomplissement, qui certes n'est pas réel, mais bien effectivement symbolique dans la cosmogonie et la syntaxe créatrice d'unité de la chose littéraire " 28 .

Dans le parcours narratif général du roman les chansons sont ainsi convoquées à des moments décisifs de transformation qui

26 Ibid., p. 250.

27 Ibid., p. 253.

28 Hermann Broch, Création littéraire et connaissance, Paris, Gallimard, 1966, p. 241. 
108

coïncident avec le parcours narratif du couple Doguicimi-Toffa. Ainsi, aux quatre phases du parcours narratif général de l'histoire de la guerre sont associés les textes de chanson et les quatre phases du parcours narratif de l'amour entre Doguicimi et Toffa. D'abord, au projet de guerre et à l'appel à la vigilance du roi, correspond l'inquiétude de Doguicimi et Toffa. Aux récits de l'oracle, de l'entêtement du roi et de la défaite du Dahomey, correspondent ensuite les récits de l'absence de Toffa, de la cour de Vidaho à Doguicimi et aux tourments de celle-ci. À la cérémonie de purification et à la mobilisation pour la revanche correspond l'attente angoissée de Doguicimi. Finalement, à la défaite des Mahinous et à la parade des vainqueurs correspond la mort de Doguicimi. Ainsi le parcours narratif du couple Doguicimi-Toffa progresse en sens opposé du parcours général du roman: la guerre des Dahoménous contre les Mahinous. La chanson, comme parole rapportée, a une fonction de mimésis qui renforce et accrédite le pouvoir du narrateur extradiégétique dont la distance donne un ton objectif au récit. La dimension anthropologique du texte de chanson apparaît aussi dans les nombreuses ellipses qui émaillent le récit, là où le lecteur s'attendait à une condamnation des fautes du roi ${ }^{29}$. Ces silences participent à la fonction idéologique du roman - garder le secret d'État - et au code de la vraisemblance - le narrateur entend dire vrai par ses silences. En plus, ces textes de chanson ont une même fonction narrative de ralentissement et de suspense dans le déroulement de l'intrigue. De la même manière, ils participent à la relance de l'histoire. Et la chanson rappelle ainsi le chour du théâtre antique.

À partir de l'hérö̈ne - Doguicimi - le roman suspend les valeurs - bien-mal, bon-méchant - par leur confrontation sans qu'une position claire n'émerge à la fin de l'œuvre. En ce sens, Doguicimi se situe dans une tradition de la littérature orale africaine dont elle applique les schémas du conte dont les séquences narratives sont scandées par le chant et l'enrichit en intégrant de nouvelles techniques d'écriture: celles du roman historique. C'est en étudiant cette rencontre de l'oralité africaine et les modes du roman européen ${ }^{30}$ que le roman prend position sur ce qu'est

29 Paul Hazoumé, op. cit., p. 176.

30 Par ce terme emprunté à Milan Kundera, je désigne un type de roman transnational dont l'entreprise historique est née avec Rabelais et Cervantes. Le "roman africain" participe de ce contexte historique du roman européen. 
l'écriture romanesque. L'analyse montre que le roman ne prend position pour aucun modèle. Leur fusion semble être un point de vue privilégié dans sa façon de juger le procès littéraire. "Le procès d'écriture est générique en ce sens que tout écrivain conçoit sa tâche en fonction de sa propre culture littéraire [...] Tout écrivain inscrit son travail dans une tradition donnée, et l'on peut mesurer parfaitement ses réalisations dans les termes mêmes de la tradition où ce travail s'inscrit " ${ }^{31}$. Le mode fictionnel de Doguicimi est chargé de valeurs qui offrent un point de vue privilégié par le roman. Celui-ci est à la fois une tragédie, qui offre des êtres héroïques dans un monde qui donne sens à leurs actes. C'est le cas de la princesse Doguicimi qui reste fidèle à son mari et refuse les avances des autres princes alors que son mari est mort. Mais Doguicimi reste, à bien des égards très ambiguë. Sa prise de parole contre l'aristocratie n'en fait pas une révolutionnaire, car, par ailleurs, elle prône la soumission absolue de la femme à l'égard de son mari ${ }^{32}$. Il en est de même du prince Toffa qui est mort sur le champ de bataille sans se révolter alors qu'il jugeait cette guerre déclenchée contre les Mahinous inutile et ruineuse pour les Dahoménous. Leur héroïsme fait penser au suicide. Celui-ci est-il un acte héroïque? A-t-il un sens moral? Au réalisme de ce roman, qui s'appuie sur les descriptions ethnologiques, en tant que technique romanesque, s'ajoute le mode de l'épopée tragique des cultures africaines dans l'ancien royaume du Dahomey. C'est ce mélange des genres qui affaiblit le caractère ethnologique et documentaire du roman et en fait une ceuvre réussie. Cet aspect polygénérique du roman permet de revenir sur le type d'écriture valorisé: la transgression des frontières des genres et les autres formes artistiques. L'énonciation romanesque ne navigue-t-elle pas entre les formes du roman historique, du roman d'amour, de la tragédie et du conte africain dont les parcours narratifs sont souvent rythmés par la chanson? Étudier ce

Certes, les thèmes varient d'une nation ou d'une époque à l'autre, mais les procédés formels restent circonscrits dans l'histoire de ce roman européen. Connaissant ce modèle, par la scolarisation et par les moyens de diffusion culturelle internationaux, les romanciers africains l'adaptent plus ou moins avec ingéniosité. Car, rappelle Milan Kundera, dont je partage le point de vue, "aucun individu ne peut ne pas imiter ce qui a déjà eu lieu" (Les testaments trahis, p. 22). S'il le connaît évidemment, devrais-je ajouter.

31 Robert Scholes: "Les modes de la fiction ", dans Gérard Genette (dir.), Théorie des genres, Paris, Seuil, p. 78.

32 Paul Hazoumé, op. cit., p. 400. 
texte demande qu'on le situe dans les préoccupations esthétiques du roman contemporain. Car, en faisant cohabiter les visions romantique et tragique de la vie, Paul Hazoumé s'inscrit dans la lignée d'autres romanciers. Tous les grands textes de Rabelais à Joyce ne se qualifient-ils par la multiplicité extrême des traits génériques? L'écriture littéraire n'est-elle pas un processus de transformation continuelle des genres existants?

\section{Pour conclure}

De ces trois brèves monographies, se dégagent quelques éléments notables pour l'enseignement du roman africain. (1) La suspension du point de vue moral, qui caractérise des actions des personnages de ces textes les inscrit dans les grandes préoccupations esthétiques du genre romanesque de Rabelais à Chamoiseau. Étudier comment la narration romanesque opère une telle suspension du jugement moral et souligner le caractère intergénérique des traits narratifs du roman moderne semblent être deux voies pertinentes pour renouveler la lecture des textes africains et faire sortir la critique des sentiers battus des thèmes de la colonisation, de la tradition et de la modernité, du village et de la ville, de la polygamie, etc. (2) En effet, l'enseignement par le genre permet de mesurer la capacité du roman à transformer, à réutiliser d'autres genres littéraires et à les intégrer à ses propres modes et d'apporter un éclairage nouveau sur le double contexte de la littérature africaine et de la littérature contemporaine, tous deux marqués dans une certaine mesure par l'esthétique polygénérique, c'est-à-dire une forme synthétique de plusieurs genres, non seulement entre les différents genres littéraires, mais aussi entre les différentes formes artistiques. (3) Réfléchir sur le fait que le roman africain participe de l'esthétique du roman contemporain a l'avantage de replacer les romans africains dans l'histoire du roman, celle de la rencontre des genres des littératures de l'Afrique et celles de l'Europe. Car, les formes du roman africain, pour ainsi dire, convoquent celles du roman européen même si les contenus peuvent différer suivant les enjeux idéologiques et sociaux. Sinon comment peut-on expliquer autrement la sève rabelaisienne qui nourrit tant de romans africains? Ce qui amène à redéfinir et à élargir le concept même de "roman africain". (4) Une telle perspective est d'autant plus importante que continuer à chercher les spécificités du roman africain débouche sur

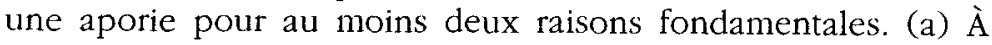


l'origine des textes que la critique regroupe sous le terme générique de "roman africain", il y a un genre qui ne cesse de se transformer et de s'enrichir de nouvelles formes: c'est le roman européen dont les traits génériques actuelles remontent à Rabelais. (b) En plus, la vie culturelle du $x^{e}$ siècle, caractérisée par plusieurs modes et moyens de communication mettant en contact diverses formes d'expression culturelle et la capacité du genre romanesque à transformer et à phagocyter toutes les formes artistiques contemporaines, permettent difficilement que l'on puisse imaginer l'existence des formes spécifiques au roman national: africain, français, latino-américain ou québécois. 